\title{
LAS CRIPTODIVISAS COMO MEDIO DE PAGO Y EL DERECHO INTERNACIONAL PRIVADO*
}

\section{CRYPTOCURRENCIES AS A MEANS OF PAYMENT AND PRIVATE INTERNATIONAL LAW}

\author{
RAÚL RUIZ RODRÍGUEZ \\ Becario de Investigación en el Área de Derecho Internacional Privado \\ Universidad de Alicante
}

Recibido: 19.06.2020 / Aceptado: 01.07.2020

DOI: https://doi.org/10.20318/cdt.2020.5628

\begin{abstract}
Resumen: el objeto del presente estudio es el análisis de la naturaleza jurídica de las denominadas criptodivisas o monedas virtuales y de las posiciones defendidas por distintos autores en base al estado normativo actual y a las decisiones emitidas por autoridades internacionales, haciendo especial hincapié en la concepción de las criptodivisas como "medio de pago" y "bienes susceptibles de propiedad". Además, se realiza también un examen acerca de la suficiencia de los mecanismos legales existentes en materia de Derecho internacional privado para lidiar con las consecuencias derivadas de dichas calificaciones y acerca de la posibilidad o necesidad de realizar modificaciones legislativas para lograr una regulación satisfactoria de las monedas virtuales.
\end{abstract}

Palabras clave: criptodivisas, blockchain, derecho internacional privado, contratación internacional.

Abstract: the purpose of this study is the analysis of the legal nature of the so-called crypto-currencies or virtual currencies and of the positions defended by different authors based on the current normative state and the decisions issued by international authorities, with special emphasis on the conception of cryptocurrencies as a "means of payment" and "property". In addition, an examination is also made of the adequacy of existing legal mechanisms in the field of private international law to deal with the consequences arising from such qualifications and of the possibility or need for legislative amendments to achieve satisfactory regulation of virtual currencies.

Keywords: cryptocurrencies, blockchain, private international law, international contracts.

Sumario: I. Introducción. II. La tecnología “blockchain” y el Derecho internacional privado. 1. El carácter esencialmente internacional de la tecnología "blockchain”. 2. Aplicaciones de la tecnología "blockchain" y Derecho internacional privado. A) "Smart contracts". B) Organizaciones descentralizadas autónomas (DAO). C) Registros. D) Tokenización o títulos-valores. E. Criptodivisas. III. Las criptodivisas y sus diversas calificaciones jurídicas en el Derecho material interno. 1. La imposibilidad de calificar las criptodivisas como moneda de curso legal. 2. Las criptomonedas como títulos-valor o activos financieros y la inviabilidad de dichas clasificaciones. 3. Clasificación de las criptodivisas como bienes susceptibles de propiedad. 4. Criptodivisas como medio de pago. IV. Calificación de las criptomonedas como medio de pago a los efectos del Derecho internacional privado. 1. Competencia judicial internacional: pago mediante criptomonedas y determinación del forum executionis. 2. Ley aplicable: pago mediante criptomonedas y art. 4 del Reglamento Roma I. V. Conclusiones.

* Agradezco al profesor Aurelio López-Tarruella su ayuda y comentarios al presente trabajo. 


\section{Introducción}

1. La denominada tecnología "blockchain" ("cadena de bloques", en nuestro idioma) se ha convertido, por méritos propios, en uno de los principales retos para los investigadores del siglo XXI. Especialistas de todos los ámbitos han centrado sus esfuerzos en estudiar las diversas aplicaciones de esta tecnología en el sector público y privado, y la ciencia del Derecho no ha sido una excepción: la ausencia de una regulación específica de dicha tecnología ha suscitado un amplio catálogo de incógnitas que van desde el valor probatorio de los registros generados por la cadena de bloques hasta la posible validez de los negocios jurídicos celebrados a través de esta ${ }^{1}$.

2. Como se explica en el apartado II, el carácter ubicuo y deslocalizado que presenta el "blockchain" provoca que las relaciones jurídicas que tienen lugar a través de esta tecnología sean, por definición, internacionales. Esta circunstancia obliga a analizar si la normativa de Derecho internacional privado (en adelante, "DIPr") está lo suficientemente preparada para regular las relaciones privadas internacionales que pueden aparecer como consecuencia de la utilización de aplicaciones basadas en la tecnología "blockchain". En particular, es preciso examinar si las normas sobre competencia judicial internacional y Derecho aplicable ofrecen a las empresas que quieren utilizar esta tecnología la seguridad jurídica necesaria para facilitar su puesta en marcha a nivel mundial.

Del análisis de los mecanismos legales existentes en la actualidad puede inferirse que, a grandes rasgos, el Derecho se encuentra lo suficientemente preparado como para lidiar con esta nueva tecnología, no siendo necesaria una reforma íntegra del marco normativo actual para otorgar una regulación satisfactoria a la cadena de bloques ${ }^{2}$. Como defendemos en el apartado II, la posibilidad que otorgan las normas de DIPr de elegir los tribunales competentes y el Derecho aplicable al negocio jurídico contribuye a eludir los problemas que la falta de adaptación de ciertos Derechos internos puede suponer para los usuarios de esta tecnología ${ }^{3}$.

3. No obstante, hay una cuestión que plantea problemas particulares para la aplicación de las normas de DIPr, como es la utilización de las criptodivisas como medio de pago. Estos problemas, conforme a lo expuesto en el apartado III, están relacionados con las dificultades con las que se han encontrado las autoridades competentes a la hora de regular esta figura, y que han derivado en la atribución de una naturaleza jurídica diferente de un ordenamiento a otro. Estas diferencias pueden generar problemas a la hora de determinar las normas de competencia judicial internacional y de Derecho aplicable que deben regir aquellos contratos que tengan como objeto la adquisición de criptodivisas y, sobre todo, aquellos en los que el pago deba llevarse a cabo mediante este mecanismo. A estas cuestiones dedicaremos el apartado IV.

\section{La tecnología “blockchain” y el Derecho internacional privado}

\section{El carácter esencialmente internacional de la tecnología "blockchain"}

4. La tecnología "blockchain" es, en palabras de C. TuR FÁunDEZ4, "una base de datos apoyada en tecnología peer to peer y por tanto compartida por múltiples nodos, en la que se registran bloques de información".

${ }^{1}$ S. Nascimento (ed), A. Pólvora (ed), A. Anderberg, E. Andonova, M. Bellia, L. Calès, A. Inamorato dos Santos, I. Kounelis, I. Nai Fovino, M. Petracco Giudici, E. Papanagiotou, M. Sobolewski, F. Rossetti, L. Spirito, Blockchain Now And Tomorrow: Assessing Multidimensional Impacts of Distributed Ledger Technologies, Luxemburgo, Publications Office of the European Union, 2019, pp. 16-20.

2 A. S. ZimmermanN, Blockchain Networks and European Private International Law, disponible en https://conflictoflaws. net/2018/blockchain-networks-and-european-private-internationale-law/ (consultado el 14 de junio de 2020).

${ }^{3}$ G. RüHL, "Smart (Legal) Contracts, or: Which (Contract) Law for Smart Contracts?", en B. CAPPIEllo, G. Carullo (Eds.), Blockchain, Law and Governance, Springer, 2020, disponible en https://papers.ssrn.com/sol3/papers.cfm?abstract_id=3552004 (consultado el 14 de junio de 2020), p. 20-21.

${ }^{4}$ C. Tur FaúnDEZ, Smart contracts. Análisis jurídico. Madrid, REUS, 2018, p.33. 
Dicha tecnología fue desarrollada con el objetivo de crear una plataforma para la realización de operaciones con la criptomoneda "Bitcoin". Sin embargo, el potencial de esta provocó que su utilización se extendiera a otros ámbitos. En la actualidad, además de "Bitcoin", hay muchas otras plataformas que emplean la tecnología "blockchain" como, por ejemplo, "Ethereum" "Tezos" o "Neo".

Para comprender su funcionamiento, es necesario estudiar dos conceptos clave:

- En primer lugar, la información está escrita en los denominados "bloques". Cada bloque de información se conecta a su predecesor respectivo utilizando los llamados "hashes", códigos de identificación que se calculan para cada bloque individual. En consecuencia, cada bloque no sólo incluye su propio "hash" sino también el "hash" de su predecesor, quedando unidos entre sí y garantizando que la información no pueda ser manipulada una vez ha sido registrada ${ }^{9}$.

- En segundo lugar, la cadena de bloques en su integridad es almacenada de forma descentralizada por cada uno de los miembros de la red. De esta manera, cada vez que se solicita una transacción relativa a la cadena de bloques, no es procesada por un solo miembro o "nodo", sino que todos los nodos comprueban la transacción e intentan alcanzar un consenso de manera que "para que la información contenida en un bloque sea considerada válida, todos los participantes deben de estar de acuerdo" ${ }^{10}$ o, por lo menos, alcanzar el mínimo exigido por las reglas de consenso establecidas por el protocolo de esa plataforma "blockchain" en particular.

5. La cadena de bloques se configura, por tanto, como un sistema articulado "a través de redes de terminales $P 2 P$ que tiene como misión registrar todas las transacciones que se van produciendo" 11 . Las notas características de esta tecnología que tienen implicaciones desde el Derecho son la inmutabilidad, la seguridad, la descentralización y la deslocalización ${ }^{12}$ :

- Inmutabilidad y seguridad: dado que la información contenida en una plataforma "blockchain" es visible para todos los usuarios, es prácticamente imposible modificarla sin que ninguno de los otros nodos lo detecte, haciendo muy difícil alterar o suprimir dicha información (únicamente sería posible mediante el consenso de todos los usuarios o, al menos, de una mayoría del 51\%). Además, el carácter inmutable de la información, junto con el hecho de que todos los datos que se introducen en la plataforma incorporen sellado de tiempo y de que las transacciones sean visibles para todos los usuarios, otorgan a esta tecnología un alto grado de transparencia que permite aumentar la confianza depositada en la misma.

- Descentralización y deslocalización: la información se registra "en una red descentralizada, por lo que no se requiere de un organismo regulado que verifique las transacciones"13 que se

${ }^{5}$ S. NAкамото, Bitcoin: A Peer-to-Peer Electronic Cash System, disponible en https://bitcoin.org/bitcoin.pdf (consultado el 14 de junio de 2020).

${ }^{6}$ Ethereum Foundation, What is Ethereum?, disponible en https:/ethereum.org/what-is-ethereum/ (consultado el 14 de junio de 2020)

${ }^{7}$ L. M. Goodman, Tezos - a self-amending crypto-ledger White paper, disponible en https://tezos.com/static/white_paper2dc8c02267a8fb86bd67a108199441bf.pdf (consultado el 14 de junio de 2020).

${ }^{8}$ Neo Team, An Open Network for the Smart Community, disponible en https://neo.org/about (consultado el 14 de junio de 2020).

${ }^{9}$ The European Union Blockchain Observatory and Forum, Legal and Regulatory Framework for Blockchains and Smart Contracts, disponible en https://www.eublockchainforum.eu/sites/default/files/reports/report_legal_v1.0.pdf (consultado el 14 de junio de 2020), pp. 36-38.

${ }^{10}$ C. Tur FaÚNDEZ, Smart contracts. Análisis jurídico. Madrid, REUS, 2018, p.34.

${ }^{11}$ A. E. Vilalta Nicuesa, Smart legal contracts y blockchain. La contratación inteligente a través de la tecnología blockchain. Madrid, Wolters Kluwer, 2019, p. 21.

12 S. Nascimento (ed), A. Pólvora (ed), A. Anderberg, E. Andonova, M. Bellia, L. Calès, A. Inamorato dos Santos, I. Kounelis, I. Nai Fovino, M. Petracco Giudici, E. Papanagiotou, M. Sobolewski, F. Rossetti, L. Spirito, Blockchain Now And Tomorrow: Assessing Multidimensional Impacts of Distributed Ledger Technologies, Luxemburgo, Publications Office of the European Union, 2019, pp. 17-20.

13 A. Ortega Giménez, "Smart contracts" y Derecho Internacional Privado. Navarra, Thomson Reuters Aranzadi, 2019, p. 30. 
realicen en la plataforma "blockchain", sino que son los miembros de dicha plataforma los que cotejarán las operaciones celebradas en esta, sin necesidad de una autoridad central que tenga el control absoluto sobre el procesamiento de la información. Asimismo, el anonimato que otorga la tecnología "blockchain" y la posibilidad de que cualquier persona pueda formar parte de la plataforma (como regla general), deriva en una situación de deslocalización caracterizada por un total desconocimiento de la ubicación de los nodos y de la información contenida en dicha plataforma.

6. Son estos dos últimos elementos los que nos permiten afirmar que estamos ante una tecnología esencialmente internacional, circunstancia que redunda en las relaciones privadas que se llevan a cabo utilizando sus diversas aplicaciones: incluso en aquellos casos en los que los elementos "analógicos" de la relación estén conectados con un solo Estado (p. ej. una transacción celebrada por dos empresas españolas mediante un "smart contract"), la tecnología utilizada conecta la situación con una pluralidad de Estados extranjeros.

Pero, además, resulta difícil imaginar empresas que estén pensado en utilizar esta tecnología en un ámbito meramente doméstico ${ }^{14}$. Se puede presumir que cualquier entidad o conjunto de entidades con la capacidad de apostar por el desarrollo de aplicaciones basadas en esta tecnología actúan o tienen la intención de actuar a nivel internacional.

Es el caso, por ejemplo, de Alibaba ${ }^{15}$ u otras plataformas que emplean esta tecnología para facilitar la identificación de productos falsificados que se intentan comercializar a través de estos mercados en línea; o VeChain", la cual emplea "blockchain" para permitir a fabricantes asignar números de identificación únicos a sus productos y hacer un seguimiento de sus movimientos a través de la cadena de suministro desde cualquier parte del mundo. También podemos destacar otras iniciativas que han implementado esta tecnología para facilitar la celebración de contratos por parte de sus clientes, con independencia de su Estado de residencia, como por ejemplo Ulysses Contract ${ }^{17}$, a través de la cual los usuarios pueden celebrar un "smart contract" para establecer un objetivo de pérdida de peso entregando cierta cantidad de "Ether" que únicamente le será devuelto en caso de alcanzar su meta.

\section{Aplicaciones de la tecnología "blockchain" y Derecho internacional privado}

7. Son varias las aplicaciones de la tecnología "blockchain" que pueden dar lugar a relaciones privadas internacionales susceptibles de regulación por el DIPr. Ahora bien, como ya hemos avanzado, más allá de los problemas que generan las criptodivisas, entendemos que no resulta necesaria una adaptación del marco normativo del DIPr para otorgar una regulación satisfactoria a esta nueva tecnología. Para comprender mejor esta idea, es necesario analizar, de manera individual, dicha aplicaciones y su funcionamiento:

\section{A) "Smart contracts"}

8. Los "smart contracts" o contratos inteligentes pueden ser definidos como "programas informáticos que ejecutan acuerdos establecidos entre dos o más partes cuando se da una condición

\footnotetext{
${ }^{14}$ Los "smart contract" son, como regla general, contratos "standard" que las empresas están utilizando o van a utilizar a nivel mundial en sus operaciones dado el potencial comercial que poseen. Lo más frecuente, por ejemplo, será que el "smart contract" sea celebrado por un dispositivo fabricado por un desarrollador chino, instalado en un vehículo eléctrico fabricado por una empresa estadounidense que es conducido por un español que ha alquilado el vehículo a una empresa francesa en Francia. Esta situación dificulta que los desarrolladores puedan adaptar el "smart contract" a las particularidades legales de cada uno de los países en los que sus productos vayan a comercializarse.

${ }^{15}$ www.alibaba.com

16 www.vechain.org

17 https://ulyssescontract.com/
} 
programada con anterioridad. Es decir, son contratos que se ejecutan y se hacen cumplir a sí mismos de manera automática"18.

Existe la posibilidad de que sean máquinas las que celebren un "smart contract": son los denominados contratos computables u operaciones "machine to machine" ("M2M", en adelante). Se trata de "contratos que se perfeccionan y ejecutan entre máquinas directamente" y en virtud de los cuales "los dispositivos se conectan y proceden a perfeccionar nuevos y sucesivos contratos bajo ciertas circunstancias previamente acordadas por las partes involucradas en un contrato legal inteligente" 19.

9. Los "smart contracts" no se constituyen como una nueva categoría de contratos, sino que se trata de "contratos propiamente dichos desde un punto de vista jurídico, que hacen uso -total o parcialmente- de la tecnología de bloques" ${ }^{20}$. De esta manera, la tecnología "smart contract" se configura, simple y llanamente, como una nueva manera de celebrar y gestionar contratos, los cuales mantendrán su naturaleza jurídica intacta, no habiendo motivos para que las reglas actuales no se apliquen sólo porque el contrato se realice mediante un "smart contract" 21 .

En concreto, esta nueva modalidad contractual no requerirá ningún cambio extraordinario ${ }^{22}$ en relación con las normas de DIPr que regulan la forma de celebración o de ejecución de las obligaciones, habida cuenta, sobre todo, de la posibilidad que ofrecen dichas normas de escoger los tribunales competentes y el Derecho aplicable para la resolución del negocio jurídico, lo que en la mayoría de casos permitirá eludir los problemas que la falta de adaptación de ciertos Derechos internos puede suponer para esta nueva modalidad de celebración y ejecución de los contratos ${ }^{23}$.

De esta forma, tal y como señala A. Legerén-Molina, "parece posible afirmar que los caracteres que actualmente configuran el contrato inteligente no parecen exigir un nuevo Derecho de contratos, siendo perfectamente aplicables -con sus adaptaciones y modulaciones- las normas actualmente existentes" ${ }^{24}$. A nuestro modo de ver, la conclusión es aplicable a la normativa de DIPr en materia de contratos.

\section{B) Organizaciones descentralizadas autónomas (DAO)}

10. Se entiende por organización descentralizada autónoma ("Decentralized Autonomous Organization", en adelante "DAO") “una empresa u organización cuyas decisiones se toman electrónicamente mediante un código de ordenador escrito o mediante el voto de sus miembros. En esencia, es un sistema de reglas codificadas que definen qué acciones tomará una organización. Es considerado por muchos como la forma más compleja de smart contract. El código del smart contract posee los estatutos de la DAO, mediante complejas reglas de gobernanza." ${ }^{25}$

${ }^{18}$ A. Ortega Giménez, "Smart contracts" y Derecho Internacional Privado. Navarra, Thomson Reuters Aranzadi, 2019, p. 29.

${ }^{19}$ A. E. Vilalta Nicuesa, Smart legal contracts y blockchain. La contratación inteligente a través de la tecnología blockchain. Madrid, Wolters Kluwer, 2019, p. 42-43.

20 A. E. Vilalta Nicuesa, Smart legal contracts y blockchain. La contratación inteligente a través de la tecnología blockchain. Madrid, Wolters Kluwer, 2019, p. 41.

${ }^{21}$ UK JuRisdition TASKForCE, "Legal statement on cryptoassets and smart contracts", The LawTech Delivery Panel, 2019, disponible en https://35z8e83m1ih83drye280o9d1-wpengine.netdna-ssl.com/wp-content/uploads/2019/11/6.6056_JO_Cryptocurrencies_Statement_FINAL_WEB_111119-1.pdf (consultado el 14 de junio de 2020), p. 32.

22 A. S. ZimmermanN, Blockchain Networks and European Private International Law, disponible en https://conflictoflaws. net/2018/blockchain-networks-and-european-private-internationale-law/ (consultado el 14 de junio de 2020).

${ }^{23}$ G. RÜHL, "Smart (Legal) Contracts, or: Which (Contract) Law for Smart Contracts?", en B. Cappiello, G. Carullo (Eds.), Blockchain, Law and Governance, Springer, 2020, disponible en https://papers.ssrn.com/sol3/papers.cfm?abstract_ id=3552004 (consultado el 14 de junio de 2020), p. 20-21.

${ }^{24}$ A. Legerén-Molina, "Los contratos inteligentes en España”, Revista de Derecho Civil, vol. V, núm. 2, abril-junio 2018, pp. 193-241.

${ }^{25}$ B. Nogales, ¿Qué es una DAO? - Organización autónoma descentralizada, disponible en https://bitcoin.es/criptomonedas/que-es-un-dao-organizacion-autonoma-descentralizada/ (consultado el 14 de junio de 2020). 
11. Si bien la nomenclatura utilizada podría apuntar a un nuevo tipo de sociedad mercantil, en realidad una DAO es fácilmente reconducible a las categorías contractuales tradicionales ${ }^{26}$ por lo que, según se ha dicho en el apartado anterior, no parecen existir problemas para aplicar la normativa sobre DIPr en la materia. De esta manera, si la voluntad de los integrantes de la DAO fuera crear una sociedad mercantil, deberían encontrar acomodo en alguna de las categorías ya existentes en las leyes nacionales sobre sociedades mercantiles, lo cual determinaría su régimen a efectos de DIPr.

\section{C) Registros}

12. Otro de los posibles usos de la tecnología "blockchain" reside en el sector registral. Son numerosos los países que han empezado a desarrollar proyectos destinados a la aplicación de esta tecnología en registros como, por ejemplo, el de la propiedad o el mercantil.

En España, sin ir más lejos, podemos destacar a Alastria ${ }^{27}$, una asociación sin ánimo de lucro que apuesta por el desarrollo de la tecnología "blockchain" y que está diseñando, junto al Colegio de Registradores de España ${ }^{28}$, métodos que permitan agilizar la gestión de todos los documentos tramitados mediante dicho colegio.

Otro claro ejemplo de aplicación del "blockchain" a un registro de la propiedad es el caso de Suecia. La administración sueca ha logrado una digitalización completa del registro de la propiedad de tierras (conocido como Lantmäteriet), facilitando la tramitación de los procesos de registro y reduciendo la duración del procedimiento de entre tres y seis meses a horas ${ }^{29}$.

Igualmente, tal y como señala A. ORTEGA GimÉNEZ ${ }^{30}$, tanto en Dubai como en Japón se están desarrollado plataformas destinadas a unificar el registro de propiedades urbanas y rústicas mediante la tecnología de la cadena de bloques, "lo que permitiría contar con una base de datos abierta en la que se pudieran consultar los datos de los 230 millones de fincas y 50 millones de edificios que se estime existen en el país asiático".

13. La cristalización de estos proyectos requerirá, en cualquier caso, el refrendo legal de estos registros, lo cual no afectará de ningún modo a las normas de DIPr sobre la materia, como por ejemplo la competencia exclusiva del artículo 24.3 del Reglamento Bruselas I bis ${ }^{31}$ en materia de registros públicos: aunque los datos estén deslocalizados, el registro público sigue estando referido a propiedades ubicadas en un territorio estatal.

\section{D) Tokenización y títulos valores}

14. El "token" puede ser definido como "una unidad de valor que una organización [o entidad privada] crea para gobernar su modelo de negocio y dar más poder a sus usuarios para interactuar con

${ }^{26}$ UK JURISDITION TASKFORCE, "Legal statement on cryptoassets and smart contracts", The LawTech Delivery Panel, 2019, disponible en https://35z8e83m1ih83drye280o9d1-wpengine.netdna-ssl.com/wp-content/uploads/2019/11/6.6056_JO_Cryptocurrencies_Statement_FINAL_WEB_111119-1.pdf (consultado el 14 de junio de 2020), p. 34.

27 Alastria, Asociación, disponible en https://alastria.io/asociacion (consultado el 14 de junio de 2020).

${ }^{28}$ Colegio de Registradores de España, Los registradores y el consorcio Alastria explorarán las posibilidades de la tecnología blockchain, disponible en http:/www.registradores.org/los-registradores-y-el-consorcio-alastria-exploraran-lasposibilidades-de-la-tecnologia-blockchain/ (consultado el 14 de junio de 2020).

${ }^{29} \mathrm{~J}$. C. AlCAIDE, Blockchain para registro de la propiedad: países pioneros en su uso, disponible en https://blog.enzymeadvisinggroup.com/blockchain-registro-propiedad (consultado el 14 de junio de 2020).

30 A. Ortega Giménez, "Smart contracts" y Derecho Internacional Privado. Navarra, Thomson Reuters Aranzadi, 2019, p. 32.

${ }^{31}$ Reglamento (UE) no 1215/2012 del Parlamento Europeo y del Consejo, de 12 de diciembre de 2012, relativo a la competencia judicial, el reconocimiento y la ejecución de resoluciones judiciales en materia civil y mercantil (en adelante, "Reglamento Bruselas I bis" o "RBI bis"), DOUE L 351/1, de 20 diciembre 2012. 
sus productos, al tiempo que facilita la distribución y reparto de beneficios" ${ }^{\prime 32}$. Estas unidades de valor se diferencian de las criptodivisas en la variedad de usos que admiten: mientras que la función principal de las monedas virtuales es la de constituirse como un medio de pago generalizado, los "tokens" servirán para aquello que las personas o entidades encargadas de su diseño y desarrollo decidan (ya sea para pagar un derecho, como incentivo o como vía de acceso a un servicio extra, etc. $)^{33}$

15. De entre todas estas funciones, podemos destacar la denominada tokenización de activos, entendida como la representación de "un derecho (personal o real, o sobre un bien tangible o intangible) en un registro distribuido (blockchain) privado a efectos legales (...) materializándose dicha representación en anotaciones contables unitarias llamadas tokens" 34 . Bajo este supuesto, la función de los "tokens" podría ser asimilada a lo de los títulos valores, documentos "que otorgan derechos privados a su poseedor y cuyo ejercicio y transmisión dependen de la tenencia del propio documento" $35 \mathrm{y}$ con los que comparten características tales como la existencia de un título representativo y un derecho subyacente.

16. La representación de un título valor a través de medios electrónicos o digitales no supone a grandes rasgos un impedimento para la aplicación de las normativa sustantiva en la materia dada la equiparación que suele darse entre documentos físicos y digitales (siempre y cuando cumplan con los requisitos formales pertinentes) por aplicación del principio de equivalencia funcional ${ }^{36} \mathrm{y}$ la posibilidad que otorga la tecnología "blockchain" de compartir, validar y verificar documentos de manera segura. Prueba de ello es la promulgación, por parte del legislador francés ${ }^{37}$, de diversos textos legales reconociendo la posibilidad de utilizar la tecnología "blockchain" 38 para la emisión, registro y transferencia de valores no admitidos a negociación.

17. En este mismo sentido, en principio, no parecen existir mayores problemas para establecer que, de acuerdo con el art. 10.3 de nuestro Código Civil ${ }^{39}$, la ley aplicable a la emisión de los "tokens" será la del Estado donde está establecida la empresa que los emite.

\section{E) Criptodivisas}

18. Otra de las principales aplicaciones derivadas de la tecnología "blockchain" son las criptodivisas o moneda virtuales, definidas por el Banco Central Europeo como "representaciones digitales

\footnotetext{
${ }^{32}$ W. MougaYar, Tokenomics - A Business Guide to Token Usage, Utility and Value, disponible en https://medium.com/@ wmougayar/tokenomics-a-business-guide-to-token-usage-utility-and-value-b19242053416\#: :text=In\%20the\%20business\%20realm\%2C\%20we,to\%20all\%20of\%20its\%20stakeholders. (consultado el 14 de junio de 2020).

${ }_{33}$ BBVA Communications, Qué es un 'token'y para qué sirve, disponible en https://www.bbva.com/es/que-es-un-token-ypara-que-sirve/ (consultado el 14 de junio de 2020).

${ }^{34}$ J. Pascual Maldonado, Tokenización de activos: naturaleza jurídica del token y del activo, disponible en http://www. legaltoday.com/firmas/legaltech/tokenizacion-de-activos-naturaleza-juridica-del-token-y-del-activo\# (consultado el 14 de junio de 2020).

${ }^{35}$ Wolters Kluwer, Títulos valores, disponible en https:/guiasjuridicas.wolterskluwer.es/Content/Documento.aspx?par ams=H4sIAAAAAAAEAMtMSbF1jTAAAUMTYzMLtbLUouLM_DxbIwMDCwNzA7BAZlq1S35ySGVBqm1aYk5xKgD1 i4j3NQAAAA==WKE (consultado el 14 de junio de 2020).

${ }^{36}$ A. FurRer, L. MÜLLER, "Functional equivalence” of digital legal transactions. Tentative translation of the "Funktionale Äquivalenz» digitaler Rechtsgeschäfte - Ein tragendes Grundprinzip für die Beurteilung der Rechtsgültigkeit von Rechtsinstituten und Rechtsgeschäften im schweizerischen Recht", published on Jusletter, Juni 18, 2018, disponible en https://www.mme.ch/fileadmin/files/documents/MME_Compact/2018/180619_Funktionale_AEquivalenz.pdf(consultado el 14 de junio de 2020), p. 13.

${ }^{37}$ H. De Vauplane, V. Charpiat, "France", The Virtual Currency Regulation Review, Edición 2, septiembre 2019, disponible en: https://thelawreviews.co.uk/edition/the-virtual-currency-regulation-review-edition-2/1197581/france (consultado el 14 de junio de 2020).

${ }^{38}$ Véase también lo dispuesto por la Comisión Nacional del Mercado de Valores [Comisión Nacional del MerCado de VALORES, Criterios en relación con las ICOs, disponible en http://cnmv.es/DocPortal/Fintech/CriteriosICOs.pdf (consultado el 14 de junio de 2020)] o por autores como F. Rosales [F. Rosales, Qué es un token, disponible en https://www.notariofranciscorosales.com/que-es-un-token/ (consultado el 14 de junio de 2020)].

${ }^{39}$ Real Decreto de 24 de julio de 1889 por el que se publica el Código Civil (en adelante, "Código Civil), Gaceta de Madrid, núm. 206, de 25 de julio de 1889.
} 
de valor no emitidas por ninguna autoridad central bancaria, institución de crédito o emisor de dinero electrónico reconocido que, en ciertas ocasiones, pueden ser utilizadas como medio de pago alternativo al dinero" ${ }^{40}$. El ejemplo más claro de ello es la criptodivisa "Bitcoin", la cual fue originalmente creada por S. NAKAмото en el año $2008^{41}$ y desde entonces ha ido adquiriendo relevancia de manera gradual hasta convertirse en la moneda virtual de uso más extendido.

La falta de consenso acerca de la naturaleza jurídica de las criptodivisas y de las implicaciones derivadas para el DIPr hace que sea necesario un análisis pormenorizado de las posibles calificaciones legales de esta tecnología y de la suficiencia de los mecanismos normativos existentes para hacer frente a las mismas. En los siguientes apartados, profundizaremos en dichas cuestiones.

\section{Las criptodivisas y sus diversas calificaciones jurídicas en el Derecho material interno}

19. La aparición de "Bitcoin" en el año 2008 generó grandes expectativas en torno al potencial de la tecnología "blockchain" para la celebración de negocios jurídicos e introdujo una atrayente posibilidad, como es la de realizar pagos a través de las denominadas criptodivisas, monedas virtuales que comparten con la tecnología de la cadena de bloques características tales como la seguridad, la inmutabilidad, la ubicuidad y la descentralización.

El creciente número de criptodivisas existentes evidencia la expansión de la tecnología "blockchain" y su progresiva incorporación a la vida diaria, pudiendo referenciar numerosos ejemplos más allá de "Bitcoin", tales como el "Litecoin" ${ }^{2}$ o el "Dash"43.

20. Ahora bien, ofrecer una definición adecuada de las criptodivisas es tarea complicada, dado que no existe una concepción uniforme acerca de la configuración jurídica y legal de las mismas, ni siquiera entre las diversas autoridades supervisoras a nivel europeo ${ }^{44}$. De hecho, como vamos a analizar a continuación, las autoridades nacionales que han debido enfrentarse con problemas relacionados con las criptodivisas les han atribuido una naturaleza jurídica divergente.

Esta situación es problemática e, indirectamente, afecta a la regulación por el DIPr de aquellos contratos internacionales en los que se establezca el pago mediante esta categoría de monedas virtuales. Y ello, porque la naturaleza jurídica que se les atribuya afectará a la calificación jurídica del contrato en cuestión y, por lo tanto, a la norma aplicable para determinar la competencia judicial internacional (en particular el Reglamento Bruselas I bis) y el Derecho aplicable (Reglamento Roma ${ }^{45}$ ).

La Comisión Europea ha impulsado el estudio "EU regulatory framework for crypto-assets", cuyo objetivo principal es el de estudiar la posibilidad de crear un marco legal uniforme para el tratamiento de los criptoactivos que permita paliar la inseguridad jurídica actual. Los resultados de dicha iniciativa serán publicados en el tercer trimestre de 2020 y está previsto que se pronuncie sobre cuestiones tales como la regulación actual de los activos criptográficos en el marco de la Unión Europea o la promulgación de directrices o "guidelines" para establecer líneas generales de actuación ${ }^{46}$.

${ }^{40}$ European Central Bank, Virtual Currency Schemes - a further analysis, disponible en https://www.ecb.europa.eu/pub/ pdf/other/virtualcurrencyschemesen.pdf (consultado el 14 de junio de 2020), pp. 9-10.

${ }^{41}$ S. Naкамото, Bitcoin: A Peer-to-Peer Electronic Cash System, disponible en https://bitcoin.org/bitcoin.pdf (consultado el 14 de junio de 2020).

${ }^{42} \mathrm{https}: / /$ litecoin.org/es/

${ }^{43} \mathrm{https}: / /$ www.dash.org/

${ }_{44} \mathrm{M}^{\mathrm{a}}$. Chamorro, Aspectos jurídicos de las criptomonedas, disponible en https://blockchainintelligence.es/wpcontent/uploads/2019/04/Art\%C3\%ADculo-doctrinal_Apectos-jur\%C3\%ADficos-de-las-criptomonedas-por-M-de-laConcepci\%C3\%B3n-Chamorro-Rodr\%C3\%ADguez.pdf (consultado el 14 de junio de 2020), p. 11.

${ }^{45}$ Reglamento (CE) N N $^{\circ} 93 / 2008$ del Parlamento Europeo y del Consejo, de 17 de junio de 2008, sobre la ley aplicable a las obligaciones contractuales (en adelante, "Reglamento Roma I"), DOUE L 177/6, de 4 julio 2008.

${ }^{46}$ Puede consultarse más información acerca de esta iniciativa en el siguiente enlace: https://ec.europa.eu/info/law/betterregulation/have-your-say/initiatives/12089-Directive-regulation-establishing-a-European-framework-for-markets-in-cryptoassets (consultado el 14 de junio de 2020). 
21. Mientras tanto, existe una gran incertidumbre sobre la calificación jurídica que pueden recibir las criptomonedas. A continuación, analizamos las distintas calificaciones adoptadas por diversas autoridades y sugeridas por la doctrina. Para empezar, explicaremos las razones por las cuales no es posible equiparar estas divisas virtuales a las monedas de curso legal y cómo el consenso negativo en torno a dicha posibilidad ha dado pie a otros planteamientos académicos, algunos de los cuales abogan por calificar a estas monedas virtuales como títulos-valor o activos financieros. No obstante, la mayoría de autores se dividen en torno a dos posibles calificaciones: la concepción de las criptomonedas como bienes intangibles susceptibles de propiedad o como medio de pago. A nuestro modo de ver, a los efectos del sistema de DIPr, esta última es la calificación que lleva a resultados más satisfactorios.

\section{La imposibilidad de calificar las criptodivisas como moneda de curso legal}

22. Para precisar si las criptodivisas merecen ser calificadas como "dinero" o "moneda de curso legal", es necesario delimitar este concepto de manera específica. En este sentido, debemos acudir al informe de 2012 del Banco Central Europeo denominado "Virtual Currency Schemes", en el cual dicho organismo otorga una definición de "dinero fiduciario", conceptuándolo como "cualquier moneda de curso legal designada y emitida por una autoridad central" 47 e indicando, a su vez, que la existencia de dicha autoridad y la confianza depositada en la misma por los usuarios de la moneda se constituyen como elementos cruciales del sistema monetario.

Una de las principales características de las criptodivisas o monedas virtuales, tal y como hemos referido anteriormente, es la descentralización o inexistencia de una autoridad encargada de su funcionamiento y control. Es por ello por lo que debemos descartar la posibilidad de que las criptodivisas sean equiparadas a una moneda de curso legal o al dinero electrónico ${ }^{48}$ dado que, a día de hoy, no han sido reconocidas de manera expresa como tal por ningún organismo oficial, no son supervisadas ni emitidas por ninguna autoridad competente y no cuentan con el respaldo de España ${ }^{49}$ ni de la Unión Europea ${ }^{50}$.

23. En este sentido, es necesario destacar la advertencia paneuropea emitida por la Autoridad Europea de Valores y Mercados, la Autoridad Bancaria Europea y la Autoridad Europea de Seguros y Pensiones de Jubilación (en adelante, "las tres AE") en 2018 advirtiendo sobre el elevado riesgo derivado del uso de las monedas virtuales y subrayando que las criptodivisas actualmente disponibles son representaciones digitales de valor no emitidas ni garantizadas por bancos centrales o autoridades públicas y que, por lo tanto, "no tienen la condición jurídica de la moneda o el dinero" ${ }^{\text {51 }}$. Esta posición es respaldada por la Directiva sobre blanqueo de capitales ${ }^{52}$, la cual reitera en su art. 3 que las monedas virtuales en ningún caso podrán ostentar la calificación de moneda de curso legal o de dinero electrónico al no tratarse de una moneda establecida legalmente.

${ }^{47}$ European Central BanK, Virtual Currency Schemes, disponible en https://www.ecb.europa.eu/pub/pdf/other/virtualcurrencyschemes201210en.pdf (consultado el 14 de junio de 2020), pp. 9-10.

${ }^{48}$ El Banco Central Europeo expone también en dicho informe que la principal diferencia entre las monedas virtuales y el dinero electrónico reside en que en el primer caso la moneda que se utiliza como unidad de cuenta no tiene una contrapartida física con estatus de moneda de curso legal, mientras que en el segundo sí.

49 Conforme a la normativa existente, las criptodivisas no pueden ser consideradas una moneda de curso legal integrada en el sistema monetario internacional español (art. 3 de la Ley 46/1998 [Ley 46/1998, de 17 de diciembre, sobre introducción al euro, $B O E$ de 18 diciembre 1998]) ni tampoco dinero electrónico (art. 1 de la Ley 21/2011 [Ley 21/2011, de 26 de julio, de dinero electrónico, $B O E$ de 27 julio 2011]).

${ }^{50}$ J. C. LLopis, Bitcoin como medio de pago en la compra de bienes, disponible en http://www.notariallopis.es/blog/i/1425/73/ bitcoin-como-medio-de-pago-en-la-compra-de-bienes (consultado el 14 de junio de 2020).

${ }^{51}$ ESMA, EBA y EIPOA, Warning to consumers on the risks of Virtual Currencies, disponible en https://eba.europa.eu/ sites/default/documents/files/documents/10180/2139750/313b7318-2fec-4d5e-9628-3fb007fe8a2a/Joint\%20ESAs\%20Warning\%20on\%20Virtual\%20Currencies.pdf?retry=1 (consultado el 14 de junio de 2020), p. 1.

${ }^{52}$ Directiva (UE) 2018/843 del Parlamento Europeo y del Consejo, de 30 de mayo de 2018, por la que se modifica la Directiva (UE) 2015/849 relativa a la prevención de la utilización del sistema financiero para el blanqueo de capitales o la financiación del terrorismo (en adelante, "Directiva sobre blanqueo de capitales"), DOUE L 156/43, de 19 de junio de 2018, p. 12. 


\section{Las criptomonedas como títulos-valor o activos financieros y la inviabilidad de dichas clasifica- ciones}

24. En cuanto a la posibilidad de calificar a las monedas virtuales como títulos-valor ${ }^{53}$, esta merece ser rechazada a tenor de lo establecido por el Tribunal de Justicia de la Unión Europea (en adelante, "TJUE") en su sentencia de 22 de octubre de 2015 (asunto "Hedqvist"

Este litigio enfrentó a la Administración Tributaria Sueca (Skatteverket) y a un particular, el Sr. Hedqvist, el cual se dedicaba a través de una sociedad a la compra de "bitcoins" para su posterior reventa a particulares y empresas, obteniendo como beneficio la diferencia entre precios. Así, el Tribunal Supremo de lo Contencioso-Administrativo de Suecia (Högsta förvaltningsdomstolen) planteó al TJUE si dichas operaciones de compraventa podían ser consideradas prestaciones de servicios y, en caso afirmativo, si dichas operaciones estaban o no exentas del Impuesto sobre Valor Añadido (en adelante, "IVA"), a tenor de lo dispuesto en el artículo 135 de la Directiva del IVA

El planteamiento de estas cuestiones obligó al TJUE a pronunciarse sobre la naturaleza jurídica de las criptodivisas y sobre las posibles calificaciones legales merecidas por las monedas virtuales, tal y como expondremos en mayor profundidad en los siguientes apartados. Ahora bien, dicho Tribunal se posicionó de manera contundente en contra de la equiparación del "bitcoin" a la figura del título valor, afirmando que "la divisa virtual «bitcoin» no es ni un título que confiera un derecho de propiedad sobre personas jurídicas ni un título que tenga una naturaleza comparable" a la de los títulos valores específicamente mencionados en el artículo 135 de la Directiva del IVA (a diferencia, por ejemplo, de las anotaciones en cuenta o de los instrumentos cambiarios).

La doctrina respalda esta postura, pudiendo destacar la opinión de autores como A. LEGERÉN-MoLINA $^{56}$, quien considera que debemos prescindir de esta posible calificación al carecer las criptodivisas de reconocimiento legal y de las figuras del "deudor" y del "emisor" frente al cual debería poder ejercitarse el correspondiente derecho.

25. Resuelta dicha cuestión, se plantea también la posibilidad de calificar a las monedas virtuales como activos financieros. Sin embargo, de nuevo aquí debemos descartar esta opción, dado que la consideración de las criptodivisas como tal implicaría la existencia de un "derecho contractual u obligación a recibir efectivo u otro activo financiero de otra entidad" ${ }^{57}$, siendo que las criptodivisas tienen valor por sí mismas y no representan un derecho de cobro que requiera la contrapartida de un pasivo financiero en otra empresa ${ }^{58}$.

\section{Clasificación de las criptodivisas como bienes susceptibles de propiedad}

26. La calificación de las criptodivisas como bienes muebles intangibles y susceptibles de propiedad se constituye como la opción que más consenso genera entre autoridades y académicos a día de

${ }_{53} \mathrm{M}^{\mathrm{a}}$. Сhamorro, Aspectos jurídicos de las criptomonedas, disponible en https://blockchainintelligence.es/wpcontent/uploads/2019/04/Art\%C3\%ADculo-doctrinal_Apectos-jur\%C3\%ADficos-de-las-criptomonedas-por-M-de-laConcepci\%C3\%B3n-Chamorro-Rodr\%C3\%ADguez.pdf (consultado el 14 de junio de 2020), pp. 14-15.

${ }^{54}$ STJUE 22 octubre 2015, Hedqvist, C 264/14.

${ }_{55}$ Directiva (UE) 2016/112/CE del Consejo, de 28 de noviembre de 2006, relativa al sistema común del impuesto sobre el valor añadido (en adelante, "Directiva del IVA"), DOUE L 347/1, de 28 de noviembre de 2006.

${ }^{56}$ A. Legerén-Molina, "Retos jurídicos que plantea la tecnología de la cadena de bloques. Aspectos legales de blockchain", Revista de Derecho Civil, vol. VI, núm. 1, enero-marzo 2019, p. 212.

57 J. Pedreira MenÉndez, "La contabilización y tributación de la moneda digital (Bitcoins)", VI Encuentro de Derecho Financiero y Tributario. Documentos de Trabajo del Instituto de Estudios Fiscales, núm. 10, 2018, disponible en https://www. ief.es/docs/destacados/publicaciones/documentos_trabajo/2018_10.pdf (consultado el 14 de junio de 2020), p. 140.

${ }^{58}$ M. J. García-Torres, "Problemas en la tributación de las operaciones con Bitcoins: calificación, prueba, valoración y control de las rentas generadas", VI Encuentro de Derecho Financiero y Tributario. Documentos de Trabajo del Instituto de Estudios Fiscales, núm. 10, 2018, disponible en https://www.ief.es/docs/destacados/publicaciones/documentos_trabajo/2018_10.pdf (consultado el 14 de junio de 2020), p. 91. 
hoy. A pesar de ello, ya adelantamos que, a los efectos de los instrumentos de DIPr, no nos parece que dicha calificación sea la más adecuada para garantizar una regulación satisfactoria de las relaciones en las que se utilicen monedas virtuales.

27. La configuración de las criptodivisas como bienes muebles encuentra su fundamento en los artículos 333, 335, 337 y 345 del Código Civil. El análisis de estos preceptos permite concluir que, al tratarse de elementos no fungibles y susceptibles de propiedad privada y de apropiación, las criptomonedas cumplen con los requisitos suficientes como para optar a dicha calificación. Así pareció entenderlo el Registro Mercantil español en el año 2014 cuando permitió la constitución de una sociedad, Conffeine S.L., cuyo capital estaba integrado únicamente por "bitcoins", a los cuales se les otorgó la calificación de "aportaciones no dinerarias" 59 .

En un sentido similar se pronunció el Tribunal Supremo, en su sentencia del 20 de junio de $2019^{60}$, en la cual condenó, por un delito continuado de estafa, al administrador único de una sociedad que había firmado contratos de gestión con varias personas que le entregaron "bitcoins" para que los reinvirtiera y devolviera las ganancias obtenidas a cambio de una comisión. Nuestro Alto Tribunal consideró que el culpable tenía la intención de apoderarse de los "bitcoins" sin cumplir con sus obligaciones y le impuso, además de una pena de prisión de dos años, la obligación de abonar una indemnización por los daños y perjuicios causados a los damnificados, dada la imposibilidad de retornar los "bitcoins" sustraídos al no tratarse de un objeto material ni tener la consideración legal de dinero.

En este contexto y a efectos de estudiar una posible restitución de las citadas monedas, el Tribunal analizó la naturaleza jurídica de las mismas y llegó a la conclusión de que "el bitcoin no es sino un activo patrimonial inmaterial (...) de contraprestación o de intercambio en cualquier transacción bilateral en la que los contratantes lo acepten, pero en modo alguno es dinero, o puede tener tal consideración legal".

Este criterio es compartido por la Dirección General de Tributos (en adelante, "DGT") en su consulta vinculante V1149-18, de 8 de mayo de $2018^{61}$, en la cual un particular plantea si el intercambio de la criptodivisa "bitcoin" por la criptodivisa "iota" origina obtención de renta a efectos del Impuesto sobre la Renta de las Personas Físicas (en adelante, "IRPF"). Pues bien, aquí la DGT manifiesta, en relación con la naturaleza legal de las monedas virtuales, que las criptodivisas "son bienes inmateriales, computables por unidades o fracciones de unidades, que no son moneda de curso legal, que pueden ser intercambiados por otros bienes, incluyendo otras monedas virtuales, derechos o servicios". No obstante, debemos señalar que en algunas de sus últimas consultas vinculantes, la DGT se ha alejado de dicho criterio, tal y como expondremos más adelante.

Cabe destacar además la opinión de organismos internacionales especializados en la materia que también se han pronunciado en este mismo sentido, como por ejemplo el UK Jurisdiction Taskforce, que en su declaración "Legal statement on cryptoassets and smart contracts" 62 emitida el pasado noviembre de 2019, concluyó que, conforme a la normativa existente, las criptomonedas deben ser tratadas como bienes intangibles susceptibles de propiedad.

28. Parece, por tanto, lógico que, teniendo en cuenta el estado normativo actual y la postura de autoridades tales como el Tribunal Supremo, la doctrina opte momentáneamente por calificar a las criptodivisas como bienes intangibles susceptibles de propiedad, a falta de una regulación que determine de manera concreta o específica la naturaleza jurídica de las monedas virtuales. Así, podemos señalar la posición de autores como A. Legerén-Molina, quien afirma que "la criptomoneda puede considerarse como un bien mueble de carácter digital y divisible, irrepetible y no copiable, susceptible de ser valora-

${ }^{59}$ E. HIJAS CID, "Bitcoins: algunas cuestiones jurídicas", El Notario del Siglo XXI, ENSXXI núm. 66, marzo-abril 2016.

${ }^{60}$ STS 20 junio $2019(R J 2109 / 2019)$.

${ }^{61}$ CVDGT 8 mayo 2018 (V1149-18).

${ }^{62}$ UK JURISDITION TASKForCe, "Legal statement on cryptoassets and smart contracts", The LawTech Delivery Panel, 2019, disponible en https://35z8e83m1ih83drye280o9d1-wpengine.netdna-ssl.com/wp-content/uploads/2019/11/6.6056_JO_Cryptocurrencies_Statement_FINAL_WEB_111119-1.pdf(consultado el 14 de junio de 2020),p. 7 
do económicamente" ${ }^{\text {63; }}$ académicas como M. J. GARCíA-TORRES FERNÁNDEZ, quien respalda esta opción al considerar "que la naturaleza jurídica de los Bitcoins es de bien mueble intangible" "64; o expertos como E. HIJAS CID, que de entre todas las calificaciones posibles se inclina también por considerar a las criptodivisas como un bien mueble digital ${ }^{65}$.

\section{Criptodivisas como medio de pago}

29. El origen de esta concepción se encuentra en la ya citada sentencia del TJUE de 22 de octubre de 2015 (asunto "Hedqvist" ${ }^{66}$ ), la cual gira en torno a las operaciones de compraventa de divisas y a la posible sujeción de las mismas al Impuesto sobre el Valor Añadido.

En ella, tras descartar la equiparación de las criptodivisas al dinero fiduciario, el TJUE concluye que "la divisa virtual de flujo bidireccional «bitcoin» (...) no puede calificarse de "bien corporal» en el sentido del artículo 14 de la Directiva del IVA, puesto que (...) no tiene ninguna finalidad distinta de la de ser un medio de pago". Así, "dado que los bitcoins no son divisas en sentido técnico, al no estar respaldados por ningún Estado" 67 , dicho órgano realiza una distinción entre divisas tradicionales (monedas que son medios de pago legales) y divisas no tradicionales, es decir, divisas "distintas a las monedas que son medios legales de pago" pero que podrán ser empleadas en negocios jurídicos siempre que las partes hayan aceptado su uso como medio alternativo a los medios legales y no tengan ninguna finalidad distintas de la de ser un medio de pago. Este sería el caso de las criptodivisas o monedas virtuales.

30. Esta calificación como medio de pago viene respaldada por la Directiva 2019/770 sobre contratos de suministro de contenidos y servicios digitales ${ }^{68}$, la cual en su art. 2 define el concepto de "precio" como "dinero o una representación digital de valor, pagadero a cambio del suministro de los contenidos o servicios digitales". A nuestro modo de ver, las criptomonedas pueden ser consideradas "representaciones digitales de valor" aceptadas como "método de pago" por las partes de una transacción económica, de manera que en principio quedarían englobadas dentro de la definición otorgada por dicha Directiva.

En este mismo sentido, debemos destacar también la ya citada Directiva sobre blanqueo de capitales, la cual tras la sentencia del caso Hedqvist modificó la definición otorgada a las criptodivisas para referirse a ellas como "representación digital de valor no emitida ni garantizada por un banco central ni por una autoridad pública, no necesariamente asociada a una moneda establecida legalmente, que no posee el estatuto jurídico de moneda o dinero, pero aceptada por personas físicas o jurídicas como medio de cambio y que puede transferirse, almacenarse y negociarse por medios electrónicos".

31. En España, esta interpretación es compartida por la DGT, la cual tras emitir varios pronunciamientos de carácter contradictorio (véase la consulta V2846-15, de 1 de octubre ${ }^{69}$, o la V1149-18,

${ }^{63}$ A. Legerén-Molina, "Retos jurídicos que plantea la tecnología de la cadena de bloques. Aspectos legales de blockchain", Revista de Derecho Civil, vol. VI, núm. 1, enero-marzo 2019, p. 211.

${ }^{64}$ M. J. García-Torres, "Problemas en la tributación de las operaciones con Bitcoins: calificación, prueba, valoración y control de las rentas generadas", VI Encuentro de Derecho Financiero y Tributario. Documentos de Trabajo del Instituto de Estudios Fiscales, núm. 10, 2018, disponible en https://www.ief.es/docs/destacados/publicaciones/documentos_trabajo/2018_10.pdf (consultado el 14 de junio de 2020), p. 91.

${ }^{65}$ E. HIJAS CID, "Bitcoins: algunas cuestiones jurídicas", El Notario del Siglo XXI, ENSXXI núm. 66, marzo-abril 2016.

${ }^{66}$ STJUE 22 octubre 2015, Hedqvist, C 264/14.

${ }_{67} \mathrm{M}^{\mathrm{a}}$. Chamorro, Aspectos jurídicos de las criptomonedas, disponible en https://blockchainintelligence.es/wpcontent/uploads/2019/04/Art\%C3\%ADculo-doctrinal_Apectos-jur\%C3\%ADficos-de-las-criptomonedas-por-M-de-laConcepci\%C3\%B3n-Chamorro-Rodr\%C3\%ADguez.pdf (consultado el 14 de junio de 2020), pp. 17.

${ }^{68}$ Directiva (UE) 2019/770 del Parlamento Europeo y del Consejo, de 20 de mayo de 2019, relativa a determinados aspectos de los contratos de suministro de contenidos y servicios digitales (en adelante, "Directiva sobre contratos de suministro de contenidos y servicios digitales"), DOUE L 136/1, de 22 de mayo de 2019, p. 17.

${ }^{69}$ CVDGT 8 mayo 2015 (V2846-18). 
de 8 de mayo, referida en el apartado anterior), ha consolidado finalmente su criterio en torno a la naturaleza jurídica de las criptomonedas a través de sus últimas consultas vinculantes.

En este sentido, podemos hacer referencia a la consulta V2034-18, de fecha 9 de julio de $2018^{70}$. El consultante es una mercantil que quiere construir una granja para el minado de criptodivisas y el desarrollo de actividades como la compraventa de monedas virtuales y desea saber la tributación de tales actividades a efectos del Impuesto sobre el Valor Añadido. En ella, la DGT analiza la naturaleza y funciones del "bitcoin" a la luz de la sentencia del TJUE de 22 de octubre de 2015 y concluye que se trata de una divisa virtual que constituye un medio de pago, por lo que las operaciones financieras vinculadas con la misma estarán exentas de IVA (conforme al art. 20.1 de la Ley 37/1992 ${ }^{71}$ y el art. 135 de la Directiva del IVA).

La DGT mantiene esta misma postura en otras consultas más recientes, como la consulta V351319 , de 20 de diciembre de $2019^{72}$, en la cual una empresa dedicada al asesoramiento legal en nuevas tecnologías desea conocer la incidencia en el IVA en el caso de que dichos servicios de asesoramiento sean facturados a sus clientes mediante monedas virtuales como el "bitcoin". Así, este órgano manifiesta que "el criterio de este Centro directivo, respecto a la transmisión de bitcoins, ha sido expuesto, entre otras, en su consulta V2034-18, de fecha 9 de julio de 2018, en donde se concluye que los bitcoins, criptomonedas y demás monedas digitales son divisas por lo que los servicios financieros vinculados con las mismas están exentos del Impuesto sobre el Valor Añadido en los términos establecidos en el artículo 20.Uno. $18^{\circ}$ de la Ley 37/1992".

32. A pesar de todo lo manifestado, esta clasificación se enfrenta a un gran obstáculo, y es que, tal y como se ha explicado en el primer apartado, no hay ningún Estado o Banco Central que avale la criptomoneda como divisa virtual o medio de pago $^{73}$. De hecho, el considerando 23 de la Directiva sobre contratos de suministro de contenidos y servicios digitales indica que "las representaciones digitales de valor también debe entenderse que incluyen las monedas virtuales, en la medida en que estén reconocidas por el Derecho nacional".

A nuestro modo de ver, el considerando llama a confusión. Ciertamente, si el precio está indicado en moneda legal, el usuario no podrá pagar en criptomoneda. Ahora bien, en la medida en que ambas partes del contrato hayan aceptado las criptodivisas como medio de pago no debería existir problema alguno para su admisión. Sería un supuesto similar a los vales o descuentos electrónicos mencionados en el mismo considerando o a los programas de canjeo de puntos por productos que utilizan muchos prestadores de servicios de Internet.

33. Está por ver si en el futuro, el pago a través de criptodivisas llegará a ser asimilado "legalmente en España y en Europa al pago mediante efectivo metálico, aunque no se le atribuya por ello la condición de dinero" ${ }^{\text {" }}$. No obstante, estamos de acuerdo con aquellos autores que entienden que el "bitcoin" podrá funcionar como mecanismo extintivo de la obligación de pago siempre y cuando las partes muestran su acuerdo expreso y voluntario ${ }^{75}$, dado que esta calificación es la que mejor se adecúa a la finalidad última de las criptodivisas.

Una vez aclarada dicha cuestión, se plantea como necesario realizar un análisis de las consecuencias derivadas para el Derecho y más concretamente, para el DIPr, de dicha calificación.

\footnotetext{
${ }^{70}$ CVDGT 9 julio 2018 (V2034-18).

${ }^{71}$ Ley 37/1992, de 28 de diciembre, del Impuesto sobre el Valor Añadido, BOE de 29 de diciembre 1992.

${ }^{72}$ CVDGT 20 diciembre 2019 (V3513-19).

${ }^{73}$ E. HiJAS Cid, "Bitcoins: algunas cuestiones jurídicas", El Notario del Siglo XXI, ENSXXI núm. 66, marzo-abril 2016.

${ }^{74}$ J. C. LLopIS, Bitcoin como medio de pago en la compra de bienes, disponible en http://www.notariallopis.es/blog/i/1425/73/ bitcoin-como-medio-de-pago-en-la-compra-de-bienes (consultado el 14 de junio de 2020).

$75 \mathrm{M}^{\mathrm{a}}$. Chamorro, Aspectos jurídicos de las criptomonedas, disponible en https://blockchainintelligence.es/wpcontent/uploads/2019/04/Art\%C3\%ADculo-doctrinal_Apectos-jur\%C3\%ADficos-de-las-criptomonedas-por-M-de-laConcepci\%C3\%B3n-Chamorro-Rodr\%C3\%ADguez.pdf (consultado el 14 de junio de 2020), pp. 17-18.
} 


\section{Calificación de las criptomonedas como medio de pago a los efectos del Derecho internacional privado}

34. Según hemos adelantado, a nuestro modo de ver, a los efectos del DIPR, las criptomonedas deben ser calificadas como un medio de pago. Esta idea se basa en tres argumentos clave:

En primer lugar, la calificación de las criptodivisas sostenida por las autoridades nacionales o por la doctrina en relación con situaciones meramente domésticas no condiciona la calificación que se le debe conceder en DIPr. Si bien los principios que informan una institución en la normativa interna inciden sobre la regulación de esa misma institución en el plano internacional, los problemas a resolver por las normas de DIPr son diferentes de los existente en las situaciones meramente domésticas ${ }^{76}$. Por consiguiente, la calificación jurídica que se otorgue a las criptomonedas en DIPr puede no coincidir con la otorgada en otras ramas del ordenamiento interno ${ }^{77}$.

En segundo lugar, la inmensa mayoría de instrumentos de DIPr existentes en materia de Derecho patrimonial son instrumentos de producción europea. Para garantizar una aplicación uniforme de esos instrumentos en todos los Estados miembros a las transacciones en las que se utilicen criptomonedas, resulta necesario sostener una calificación autónoma de esta figura ${ }^{78}$. Para ello deben tenerse en cuenta el contenido y la finalidad del instrumento europeo en concreto ${ }^{79}$.

En tercer lugar, la calificación de esta figura a los efectos del DIPr debe estar guiada por la necesidad de otorgar la regulación más satisfactoria posible a las relaciones privadas internacionales en las que se utilicen criptomonedas. A nuestro modo de ver, ello exige ofrecer una interpretación que facilite la utilización de las criptomonedas como medio de pago en el comercio internacional.

35. De acuerdo con estos tres postulados, entendemos que la calificación de las criptomonedas como medio de pago ofrece resultados más satisfactorios que otras calificaciones alternativas a la hora de determinar los tribunales competentes y la ley aplicable a las transacciones comerciales en las que se utilicen. Y ello porque, si se optara por considerar las criptomonedas como "activos intangibles", la calificación jurídica de estas transacciones dificultaría innecesariamente la aplicación del art. 7.1 del RBI bis (art. 5.1 del Convenio de Lugano ${ }^{80}$ ) y del art. 22.quinquies.a de la LOPJ $^{81}$ para determinar la competencia judicial internacional; y del art. 4 del Reglamento Roma I para establecer la ley aplicable al contrato en el que la obligación de pago se reflejara en criptomonedas.

\section{Competencia judicial internacional: pago mediante criptomonedas y determinación del forum executionis}

36. Como se ha adelantado, la utilización de tecnología "blockchain" en la contratación internacional (los llamados "smart contracts") no presenta problemas para la determinación de los tribunales

\footnotetext{
${ }^{76}$ A. López-Tarruella Martinez, Manual de Derecho internacional privado (3 ${ }^{a}$ Ed), Alicante, ECU, 2017, p. 33.

77 En el mismo sentido, en relación con el Derecho inglés se pronuncia M. NG [M. NG, "Choice of law for property issues regarding Bitcoin under English law”, Journal of Private International Law, vol. 15, núm. 2, septiembre 2019, pp. 321]

${ }^{78}$ Ejemplo de ello es la citada sentencia del TJUE de 22 de octubre de 2015 [STJUE 22 octubre 2015, Hedqvist, C 264/14], en la que el Tribunal señala, con carácter preliminar que "según la jurisprudencia del Tribunal de Justicia, las exenciones contempladas en el artículo 135, apartado 1, de la Directiva del IVA constituyen conceptos autónomos de Derecho de la Unión que tienen por objeto evitar divergencias de un Estado miembro a otro a la hora de aplicar el régimen del IVA (véanse, en particular, las sentencias Skandinaviska Enskilda Banken, C-540/09, EU:C:2011:137, apartado 19 y jurisprudencia citada, y DTZ Zadelhoff, C-259/11, EU:C:2012:423, apartado 19)".

${ }^{79}$ En relación con el Reglamento Bruselas I bis, véase, por todas, la STJUE 14 febrero 2019, Milivojević, C 640/17: "los conceptos utilizados en el Reglamento (...) deben interpretarse de forma autónoma, principalmente con referencia al sistema y a los objetivos de dicho Reglamento, para garantizar su aplicación uniforme en todos los Estados miembros". En lo relativo al Reglamento Roma I, la doctrina sostiene que "los conceptos y normas del Reglamento Roma I deben tener un "significado propio», con independencia de lo que establezcan los Derechos nacionales de los Estados miembros" [A. CALVo CARAVACA, J. Carrascosa González, Derecho Internacional Privado. Volumen II, Granada, Comares, 2018, p. 944].

${ }^{80}$ Convenio relativo a la competencia judicial y a la ejecución de resoluciones judiciales en materia civil y mercantil, hecho en Lugano el 30 de octubre de 2007 (en adelante, "Convenio de Lugano" o "CL"), DOUE L 339/3 de 21 diciembre 2007

${ }^{81}$ Ley Orgánica 6/1985, de 1 de julio, del Poder Judicial (en adelante, "LOPJ”), BOE de 2 de julio de 1985.
} 
competentes para conocer de los contratos internacionales celebrados por consumidores. Ello es así porque la aplicación de los foros de competencia en la materia (arts. 17 a 19 del Reglamento Bruselas I bis) no resulta afectada por las novedades en la celebración y, en su caso, ejecución de las obligaciones que incorpora la tecnología "blockchain"82.

37. En relación con los contratos en general, en principio, no deberían existir mayores problemas para otorgar validez a cláusulas de sumisión incluidas en un "smart contract" gracias a la referencia genérica del art. 25.2 del RBIbis a los acuerdos de elección de foro celebrados por medios electrónicos ${ }^{83}$. Ahora bien, la remisión a la ley del Estado miembro cuyos tribunales han sido designados en el acuerdo para determinar su validez material puede resultar problemática por cuanto, a día de hoy, no se puede afirmar que los "smart contracts" tengan validez, como forma de manifestar el consentimiento, en todos los Estados miembros.

Siendo esto así, en aquellos supuestos en los que la cláusula de sumisión no sea válida, o cuando no se haya incluido en el contrato, la determinación de los tribunales competentes debe llevarse a cabo de acuerdo con el art. 7.1 del Reglamento Bruselas I bis / art. 5.1 CL (si el demandado está domiciliado en la UE o un Estado parte del Convenio de Lugano); o el art. 22.quinquies a) LOPJ (por remisión del art. 6 RBI bis / art. 4 CL, si el demandado en un tercer Estado). Y es en este punto donde aparecen los problemas si el contrato establece el pago mediante criptodivisa.

38. A la hora de aplicar el art. 7.1 del Reglamento Bruselas I bis, si se siguiera la jurisprudencia de nuestro Tribunal Supremo y se otorgara la calificación de las criptodivisas como "bienes muebles intangibles", resultaría difícil sostener la aplicación de las reglas especiales de la letra b):

- Si se tratara de una entrega de mercancías a cambios de "bitcoins" estaríamos ante una especie de permuta.

- Si se tratara de la prestación de su servicio a cambio de "bitcoins", ante un contrato de difícil calificación pero, sin duda, no se estaría ante el tradicional esquema contractual de prestación de servicios a cambio de un precio.

En consecuencia, no siendo aplicables ninguna de las reglas especiales, sería preciso acudir a la regla general de la letra a), con las dificultades que ello entraña: resultaría preciso identificar la obligación concreta cuyo cumplimiento se reclama; y, posteriormente, fijar el lugar donde debía cumplirse. De no venir éste establecido en el contrato, debería determinarse a partir de la lex contractus $^{84}$ que, como veremos, también presenta problemas para su identificación.

39. Por su parte, si el demandado estuviera domiciliado en un tercer Estado, determinar si la obligación contractual debía cumplirse en territorio español de acuerdo con el art. 22.quinquies a) de la LOPJ tampoco estaría exento de problemas. Si la obligación incumplida fuera el pago mediante criptodivisas, entendida como "bien mueble intangible", ¿dónde debería entenderse que debía realizarse la entrega? Tratándose de una tecnología que goza del atributo de "deslocalización", difícilmente podría sostenerse que ese lugar fuera España.

${ }^{82}$ M. Cervera Martínez, “Aproximación a la protección del consumidor en los nuevos contratos inteligentes o smart contracts", Actualidad Civil, núm. 6, junio 2019, disponible en http:/www.icablex.es/Content/documento.aspx?param$\mathrm{s}=$ H4sIAAAAAAAEAMtMSbF1CTEAAiNLMyMTc7Wy1KLizPw827DM9NS8k1S13MSSktQiWz9HAIhze_QqAAAAWKE (consultado el 14 de junio de 2020).

${ }^{83}$ El párrafo segundo del artículo 25 establece que "se considerará hecha por escrito toda transmisión efectuada por medios electrónicos que proporcione un registro duradero del acuerdo". Como se ha indicado anteriormente, una de las principales características de los contratos inteligentes es la inmutabilidad, por lo que, en principio, la cláusula de sumisión podrá insertarse en el "smart contract" de manera válida y sin mayores dificultades, dando así cumplimiento al requisito de celebración del acuerdo por escrito.

${ }^{84}$ En este sentido, véase la sentencia del TJUE del 14 de marzo de 2013 [STJUE 14 marzo 2013, Feichter, C 419/11] 
40. La calificación de las criptomonedas como medio de pago permite llegar a resultados más adecuados por cuanto el contrato del que traen causa no sufre una modificación en su calificación. Así, cuando el demandado estuviera domiciliado en un Estado miembro, si se utilizara "bitcoin" para pagar una prestación de servicios (p. ej. un servicio digital como el alojamiento de datos, de SaaS ${ }^{85}$ o, incluso, un servicio tradicional), se trataría de un contrato regulado por la regla especial del art. 7.1 b) del RBI bis.

Esta calificación está en consonancia con lo sostenido por A. CALVO CARAVACA Y J. CARRASCOSA GonZÁlez, para quienes en los contratos de prestación de servicios "la remuneración que recibe el prestador del servicio no debe ser necesariamente una "cantidad dineraria»" ${ }^{86}$ sino que toda "ayuda" que suponga un valor económico para el prestador del servicio podrá ser considerada como remuneración, a tenor de lo establecido por el TJUE en resoluciones como la Sentencia de 14 de julio de 2016 (asunto "Granarolo" $\left.{ }^{\prime 7}\right)$. En ella, dicho órgano judicial, al pronunciarse sobre la posible existencia de una relación contractual entre una empresa francesa y una italiana y la naturaleza de la misma, concluye que "la remuneración atribuida como contrapartida de una actividad (...) no se puede entender en el sentido estricto de pago de una cantidad dineraria. La redacción muy general del artículo 5, punto 1, letra b), segundo guion, del Reglamento Bruselas I [actual art. 7.1 del RBI bis] no exige tal restricción".

De hecho, como afirman los referidos autores, "el TJUE no ha exigido [en ninguna sentencia hasta la fecha] para calificar un contrato como una prestación de servicios que exista una remuneración o pago en favor del prestador del servicio. Solo se exige que éste realice una «actividad positiva». En consecuencia, la prestación gratuita de servicios debe ser calificada como un contrato de prestación de servicios" 88 .

No parece que existan problemas para extender esta interpretación a los contratos en los que se compran mercancías a cambio de un pago en criptomonedas. De esta forma, no estaríamos ante un contrato de permuta, sino ante un contrato internacional de compraventa de mercaderías en el que el pago se lleva a cabo mediante criptomoneda, por lo que la regla especial del art. 7.1 b) RBI bis resultaría aplicable.

41. En definitiva, la calificación de la criptomoneda como medio de pago implica que los contratos de compraventa de mercaderías y de prestación de servicios no verán alterada su calificación jurídica, por lo que continuarán siendo aplicables las reglas especiales del art. 7.1 b), las cuales resultan más fáciles de aplicar en la práctica que la regla general del art. 7.1 a). Así, en los contratos de compraventa de mercancías, la jurisdicción correspondería a los tribunales del Estado de entrega de las mercancías ${ }^{89}$ $\mathrm{y}$ en los de servicios, a los del lugar de prestación del servicio ${ }^{90}$.

42. Por último, en relación con el art. 22.quinquies.a) de la LOPJ, la consideración de la criptomoneda como medio de pago permitiría interpretar que el lugar de cumplimiento de la obligación está referido a la obligación característica que, en este caso, sería la no dineraria. Es decir, la competencia de los tribunales españoles estaría justificada si la entrega de la cosa, o la prestación del servicio debiera llevarse a cabo en territorio español.

\section{Ley aplicable: pago mediante criptomonedas y art. 4 del Reglamento Roma I}

43. Al igual que ocurre en relación con el Reglamento Bruselas I bis, la utilización de tecnología "blockchain" en los contratos celebrados por consumidores no exige ningún esfuerzo interpretativo a la hora de determinar la ley aplicable. Y ello porque ni los criterios de conexión (residencia habitual del consumidor y autonomía conflictual condicionada) ni las condiciones de aplicación del art. 6 del Reglamento Roma I se ven afectados por dicha tecnología.

\footnotetext{
85 "Software as a Service", contratos en virtud de los cuales los usuarios pueden acceder mediante Internet a aplicaciones basadas en la nube (p. ej. herramientas ofimáticas).

86 A. Calvo Caravaca, J. Carrascosa González, Derecho Internacional Privado. Volumen II, Granada, Comares, 2018, p. 925

87 STJUE 14 julio 2016, Granarolo, C 196/15.

88 A. Calvo Caravaca, J. Carrascosa González, Derecho Internacional Privado. Volumen II, Granada, Comares, 2018, p. 992.

89 En este sentido, véase la sentencia del TJUE del 25 de febrero de 2010 [STJUE 25 febrero 2010, Car Trim, C 381/08]

90 En este sentido, véase la sentencia del TJUE del 10 de septiembre de 2015 [STJUE 10 septiembre 2015, Ferho, C 47/14].
} 
44. Los problemas pueden aparecer, de nuevo, en los contratos en general, especialmente cuando las partes no hayan elegido la ley aplicable, o esta elección no resulte válida de acuerdo con el art. 3 del Reglamento Roma I. Como hemos indicado anteriormente todavía no es posible saber si los distintos ordenamientos consideran que los "smart contracts" son una modalidad válida de manifestación del consentimiento.

La determinación del Derecho aplicable a partir de los criterios subsidiarios del art. 4 puede resultar compleja si las criptomonedas se califican como "bienes muebles intangibles". Y ello porque, en tal caso, será difícil entender que el contrato está incluido en una de las categorías establecidas en el apartado 1. De nuevo, se rompe el esquema contractual tradicional de entrega de la cosa, o prestación del servicio a cambio del pago de un precio al que parece responder las letras a) y b) del art. 4.1 del Reglamento Roma I. Sería necesario acudir, por tanto, al apartado 2 y, en su caso, al apartado 4, cuya aplicación resulta más compleja para el aplicador del Derecho y no garantiza el mismo grado de seguridad jurídica que las soluciones del apartado primero.

Así, por ejemplo, si la compraventa de mercancías mediante "bitcoins" fuera calificada como un contrato de permuta, no sería posible identificar a la parte que lleva a cabo la obligación característica, por lo que la ley más estrechamente vinculada debería designarse directamente, en atención a todos los elementos que rodean el contrato ${ }^{91}$. En este sentido, autores como $\mathrm{M}$. $\mathrm{NG}^{92}$ consideran que "la naturaleza descentralizada y autónoma de la red 'Bitcoin'dificulta la aplicación de este criterio".

Por todo ello, si bien la aplicación de los apartados 2 y 4 debería llevar de igual manera a la regulación del contrato por la ley del Estado de residencia habitual del vendedor y del prestador del servicio, a nuestro modo de ver, resulta más sencillo alcanzar ese resultado si las criptomonedas son calificadas como medios de pago.

45. Efectivamente, esta aproximación a las criptomonedas conllevaría que los contratos de compraventas de mercaderías y los de prestación de servicios (fueran estos digitales o tradicionales) mantendrían su calificación jurídica y, por lo tanto, el Derecho aplicable se determinaría a partir del art. 4.1 del Reglamento Roma I. En el primer caso, también se podría sostener que el pago mediante criptomonedas no afecta a la aplicación del Convenio de Viena sobre compraventa internacional de mercaderías $^{93}$, si bien, en vista del carácter internacional del Convenio, esta interpretación puede no resultar definitiva.

\section{Conclusiones}

46. A modo de conclusión y tras el análisis realizado en torno a la naturaleza jurídica de las criptodivisas y a la suficiencia de los mecanismos legales existentes para lidiar con dicha tecnología, cabe afirmar que las normas de Derecho internacional privado actuales se encuentran lo suficientemente preparadas como para otorgar una regulación satisfactoria a los negocios jurídicos que integren las denominadas monedas virtuales, incluso en aquellos supuestos en los que llegue a producirse una recalificación del contrato derivada de la concepción predominante de las criptodivisas como bienes intangibles susceptibles de propiedad.

47. No obstante, dada la dificultad de determinar cuestiones tales como la competencia judicial o la ley aplicable en los supuestos de recalificación del contrato (y especialmente cuando este se transforma en una modalidad del contrato de permuta), consideramos que es más adecuada, a efectos de

${ }^{91}$ El TJUE, en su sentencia de 23 de octubre de 2014 [STJUE 23 octubre 2014, Haeger \& Schmidt, C 305/13] afirma que el juez nacional deberá llevar a cabo "una apreciación global de la totalidad de los elementos objetivos que caracterizan la relación contractual y apreciar el elemento o elementos que, a su juicio, son más significativos".

${ }_{92}$ M. NG, "Choice of law for property issues regarding Bitcoin under English law", Journal of Private International Law, vol. 15, núm. 2, septiembre 2019, pp. 315-338.

${ }^{93}$ Convención de las Naciones Unidas sobre los contratos de compraventa internacional de mercaderías, hecha en Viena el 11 de abril de 1980 (en adelante, “Convenio de Viena”), BOE de 13 junio 1980. 
Derecho internacional privado, la clasificación de las criptodivisas o monedas virtuales como medio de pago alternativo. Todo ello sin perjuicio de que en el futuro, tal y como parece indicar la iniciativa de la Comisión Europea mencionada anteriormente, las autoridades internacionales lleguen a un consenso en torno a la naturaleza jurídica de las criptodivisas y procedan a una modificación del marco normativo que unifique el tratamiento legal de las mismas. 